\title{
DISCRETE REPRESENTATION MODEL OF MULTIPLY CONNECTED PIPELINES WITH DISTRIBUTED PHYSICAL PROPERTIES
}

\author{
Igor Lyutak \\ Ivano-Frankivsk National Technical University of Oil and Gas, department of software of computer-based systems, \\ Karpatska str. 15, Ivano-Frankivsk, 76019, Ukraine, e-mail: ihorlt@techemail.com,www.nung.edu.ua
}

\begin{abstract}
In this paper presented approach of developing chain model which describe distributed physical properties of multiply connected pipeline. It is shown that the digital autoregressive filter can be used to analyze signals of wave propagation in the chain model.
\end{abstract}

Keywords: Pipeline, chain model, digital filter.

\section{INTRODUCTION}

In gas or oil industry, a pipe that is not connected to anything else is not much use. But a main line that's connected to a network of subsidiary pipes is powerful because it can distribute or gather product throughout city or enterprise.

To develop approaches of identification, diagnostics and prediction of pipeline systems behavior it is necessary both to use computing engineering and to elaborate models of pipeline operation. Moreover it is required not just correlation between input/output signals of measured parameters but also it is required functional statistic correlation between structure and imperfections of pipeline under investigation.

The main contribution in discrete model representation was made in paper [1]. Further development of discrete models with introduction of directional changes of its physical properties was made in paper [2]. In this paper was made an assumption that a unit which conducts a measured signal can be modeled by one lumped element which describes continuum with set of material particle. In a number of cases such approach gives reasonable degree of accuracy. But in a case when the time of signals propagation in the unit has commensurable quantities with the time delay from distinct measured parameters or imperfections this approach is unacceptable.

In the paper presented a development of structural model of variously distributed in time processes which are interrelated to each other functionally or stochastically. The novelty of the developed model is the possibility to consider both variously distributed processes and processes with accumulative changes. The main approach of the model development is to build nonstationary autoregressive filters.

\section{DISCRETE REPRESENTATION OF THE MODEL}

The basic assumption of the approach of building model lies in hypothesis that real units of dynamic systems under operation are always spatially prolonged. In addition it implies that measured signals in those units propagate in the medium. The medium is characterized by continuum of material particles. In each material particle arises own transient processes which depend not only on time but on spatial position of material particle. These processes can be deterioration, ageing, changes of environment parameters etc. The made assumption obtains a special significance while diagnosing units with distributed in space parameters.

The signals, which propagating in dynamic systems, have as a rule broad spectrum of frequencies and they cause impact on the units in system in the same broad spectrum of frequencies. Such impacts result in accumulation stresses and defects along individual units with different intensity. To calculate values of stresses and size of defects it is necessary to have both the model of signals distribution in space and the model of interrelation of units' properties of the system in space with propagating signals characteristics. 


\section{MODELLING OF SIGNALS DISTRIBUTION IN SPACE}

As our interest lies in a field of diagnostics of units in the system then we limit a scope of the research to model both processes of reversal vibrations and stresses fluctuations. Such processes cause the most rapid units wear and ageing of the system.

In general an equation of unit oscillation in the system has a form [3]:

$$
\begin{aligned}
& \rho \frac{\partial^{2} u}{\partial t}=\operatorname{div}(p \cdot \operatorname{grad}(u))- \\
& -q \cdot u+F\left(X\left(x_{1}, x_{2}, x_{3}\right), t\right),
\end{aligned}
$$

where $u$ is a function which describe oscillation of the unit in the system; $X\left(x_{1}, x_{2}, x_{3}\right)$ is coordinate system; $t$ is the time; $F\left(X\left(x_{1}, x_{2}, x_{3}\right), t\right)$ is function of external disturbance; $\rho$ is density; $p, q$ are parameters which values depend on properties of the system.

Under divergence and gradient definitions the first member of a right part of Eq. (1) can be expressed as:

$$
\operatorname{div}(p \cdot \operatorname{grad}(u))=\sum_{i=1}^{n} \frac{\partial}{\partial x_{i}}\left(p \frac{\partial u}{\partial x_{i}}\right),
$$

where $n$ can be 1, 2 and 3 and it depends on considered dimension.

Eq. (1) and Eq. (2) describe processes in the system from one theoretical and methodological point of view. To simplify these equations consider plane wave propagation. This simplification is suitable for the most technical problems inclusive pipelines. The plane wave approach reduces independent variables to two namely the time and one dimension. With two independent variables Eq. (1) describes one-dimensional harmonic motion. Solving Eq. (1) with $F(x, t) \neq 0$ leads to determination of forced oscillation. Solving Eq. (1) with $F(x, t)=0$ leads to determination of free oscillations. General solution of Eq. (1) can be expressed as:

$$
u(t, x)=u^{-}(x+c t)+u^{+}(x-c t),
$$

where $u^{-}, u^{+}$are doubly continuously differentiable functions.

Function $u^{+}(x-c t)$ describes disturbance from a point $x_{0}$ in the time moment $t_{0}$ to a point $x=x_{0}+c t$ in the time moment $t$. In other words this function describes a wave which propagates from the left side of the model (point $x_{0}$ ) to the right with velocity $c$.
Similarly function $u^{-}(x-c t)$ describes a wave which propagates from the right side of the model to the left with the same value of velocity.

For an unambiguous solution of Eq. (1) it is necessary to set both initial conditions and boundary conditions of oscillatory motions.

In a mechanical elastic unit longitudinal strains $u(x, t)$ and impulse $p(x, t)$ can be expressed as:

$$
\begin{aligned}
& \frac{\partial u(x, t)}{\partial x}=-\frac{1}{E(x) S(x)} \frac{\partial p(x, t)}{\partial t} ; \\
& \frac{\partial p(x, t)}{\partial x}=-\rho \cdot S(x) \frac{\partial u(x, t)}{\partial t}
\end{aligned}
$$

where $S(x)$ is area of crosscut of elastic unit, $E(x)$ is coefficient of elasticity of the first order.

Combining two equations (4) in one leads to

$$
\rho \cdot S(x) \frac{\partial^{2} u(x, t)}{\partial^{2} t}=\frac{\partial}{\partial x}\left(E(x) S(x) \frac{\partial u(x, t)}{\partial x}\right),
$$

Under conditions of $E(x)=$ const, $S(x)=$ const, Eq. (5) can be reduced to

$$
\frac{\partial^{2} u(x, t)}{\partial x^{2}}=\frac{1}{c^{2}} \frac{\partial^{2} u(x, t)}{\partial t^{2}},
$$

where $c=\sqrt{E(x) / \rho}$ is a velocity of deformational wave in the unit.

In a case of the real system is a multiply connected pipeline with different geometry of pipes, Fig. 1 it is necessary to consider geometry of the unit.

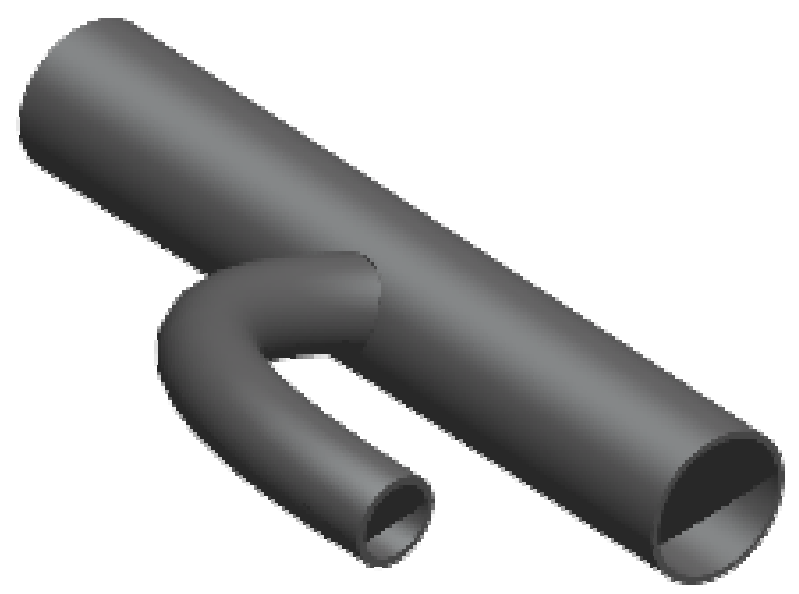

Fig. 1 Multiply connected pipeline

In this case the unit is a hollow cylinder with the air inside, Fig. 2. The real system is modeled by set of units with constant wall thickness and constant 
pressure along of the unit. Pressure component and bulk acoustic wave velocity are interrelated with each other as

$$
\begin{aligned}
& \frac{\partial P(x, t)}{\partial x}=-\frac{\rho}{S(x)} \frac{\partial u(x, t)}{\partial t}, \\
& \frac{\partial u(x, t)}{\partial x}=-\frac{S(x)}{\rho c^{2}} \frac{\partial P(x, t)}{\partial t},
\end{aligned}
$$

where $\rho$ is the density of the air, $S(x)$ is area of crosscut of hollow cylinder, $c$ is bulk acoustic wave velocity in the air.

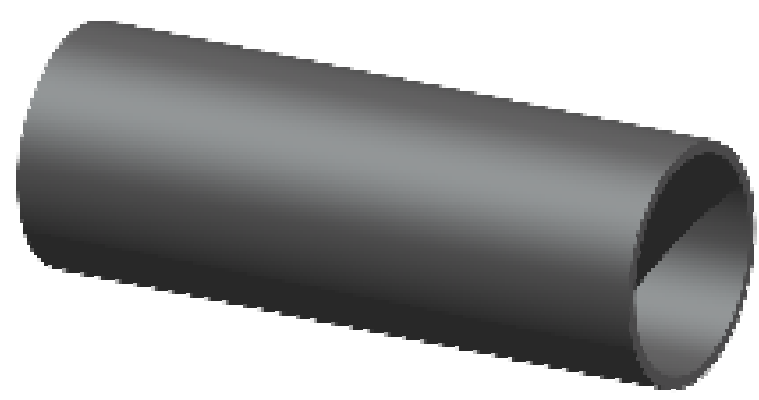

Fig. 2 The unit of pipeline model

Eq. (7) can be transformed into acoustic horn equation

$$
\frac{\partial}{\partial x}\left(\frac{1}{S(x)} \frac{\partial u(x, t)}{\partial x}\right)=\frac{1}{c^{2} S(x)} \frac{\partial^{2} u(x, t)}{\partial t^{2}} .
$$

In case of $S(x)=$ const Eq. (8) can be transformed into general wave propagation equations

$$
\left\{\begin{array}{l}
\frac{\partial^{2} u(x, t)}{\partial x^{2}}=\frac{1}{c^{2}} \frac{\partial^{2} u(x, t)}{\partial t^{2}}, \\
\frac{\partial^{2} P(x, t)}{\partial x^{2}}=\frac{1}{c^{2}} \frac{\partial^{2} P(x, t)}{\partial t^{2}} .
\end{array}\right.
$$

Let's consider the case when the unit is hollow cylinder filled with liquid (water or oil) Fig 3.

Our interest lies in observing laminar flow in the pipe unit. In this case friction of the flow to wall of the pipe and changes of the acoustic wave velocity can be neglected with respect to infinitesimally of their values. Under Zhukovsky approach we have [3]

$$
\left\{\begin{array}{l}
\frac{\partial h(x, t)}{\partial x}=-\frac{\rho(x)}{c} \frac{\partial q(x, t)}{\partial t}, \\
\frac{\partial q(x, t)}{\partial x}=-\frac{1}{c \rho(x)} \frac{\partial h(x, t)}{\partial t}
\end{array}\right.
$$

where $h(x, t)$ is relative change of hydraulic drop, $q(x, t)$ is relative change of supply rate.

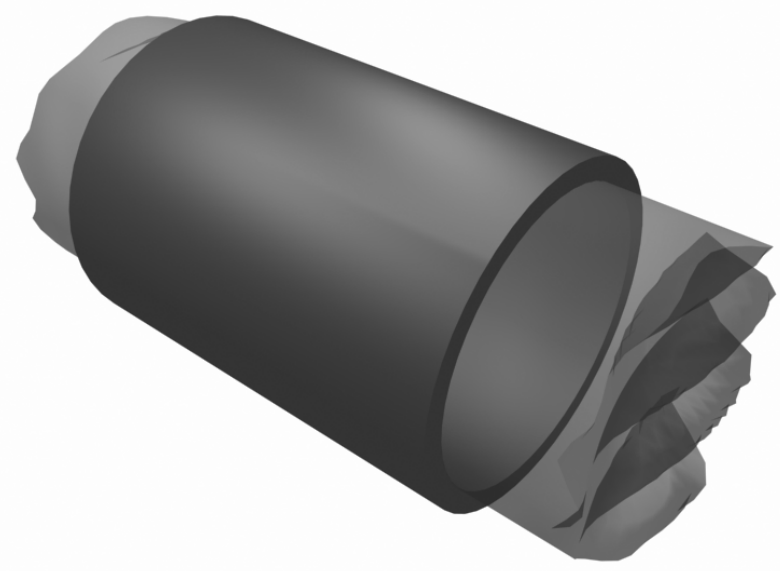

Fig. 3 Pipe unit filled with liquid

From Eq. (10) density $\rho(x)$ can be derived as

$$
\rho(x)=\frac{c Q}{g S(x) H}=\frac{c V(x)}{g H},
$$

where $g$ is the gravity, $V(x)$ is flow velocity, $Q$ is nominal value of the supply rate and $H$ is nominal value of the hydraulic drop.

Deriving wave equations for units with different shapes, we made an assumption that continuum of the points which form one unit is homogenous medium with independent from space coordinate physical properties.

In the most real practical situations this assumption fails. It is obvious that analysis of the system with this assumption becomes very complicated. To approximate non-uniformity of the unit physical properties in the space it is suitable to use approach of chain data representation. In accordance with this approach a continuous function of representation in the space non-uniformity is replaced by a discrete set of step functions. For example, one elastic unit with variable in the space hardness values is changed by set of the units with constant hardness value and the pipe with variable cross-sections is changed by set of shot pipes with constant cross-section. It makes analysis of chain model easier when the units are equal in length. A number of the units in chain model are defined with the assumption of accuracy of performed calculation.

As it implies that the units in chain model have constant physical properties along itself then we can apply Eq. (1) to every unit separately. 


\section{CHAIN MODEL ANALISYS}

Let us examine in details physical processes in the arbitrary unit with sequence number $i$. In accordance with Eq. (7) in this unit a resultant wave can be expressed as a superposition of waves $u_{i}^{+}$and $u_{i}^{-}$

$$
u_{i}(x, t)=u_{i}^{+}(t-x / c)+u_{i}^{-}(t+x / c) .
$$

The time of resultant wave propagation through the unit can be calculated as

$$
\tau=\frac{l}{c}
$$

where $l$ is a length of the unit.

Let move a center of coordinate system to the left boundary of a unit. At the left boundary waves can be expressed as

$$
u_{i}^{+}(t-0 / c)=u_{i}^{+}(t), u_{i}^{-}(t+0 / c)=u_{i}^{-}(t)
$$

Respectively at the right boundary waves can be expressed as

$$
\begin{aligned}
& u_{i}^{+}(t-l / c)=u_{i}^{+}(t-\tau) \\
& u_{i}^{-}(t+l / c)=u_{i}^{-}(t+\tau)
\end{aligned}
$$

As the chosen set of the units models the single real part of the system with distributed physical properties, then at the boundaries of the units a condition of continuity of physical properties must obey

$$
u_{i-1}(t, l)=u_{i}(t, 0)
$$

For further analysis it is necessary to get the additional information about binding parameter of main modeled real part with process of wave propagation in the unit. This information can be obtained from Eq. (4)

$$
P_{i}(x, t)=\frac{\rho \cdot c}{S_{i}}\left(u_{i}^{+}(t-x / c)+u_{i}^{-}(t+x / c)\right)
$$

where $P_{i}(x, t)$ is a pressure in the single unit.

To calculate how much wave energy is passed to the next unit and how much energy is reflected at the boundaries we introduce reflection coefficient

$$
k_{i}=\frac{S_{i}-S_{i-1}}{S_{i}+S_{i-1}} .
$$

By constituting Eq. (18) into boundary conditions (14)-(15) can be expressed wave equations as

$$
\begin{aligned}
& u_{i+1}^{+}(t)=\left(1+k_{i}\right) u_{i}^{+}(t-\tau)+k_{i} \cdot u^{-}(t), \\
& u_{i}^{-}(t)=-k_{i} \cdot u_{i}^{+}(t-\tau)+\left(1-k_{i}\right) \cdot u_{i+1}^{-}(t) .
\end{aligned}
$$

Eq. (19) corresponds to flowchart of Kelly Lockbaum [4], (Fig. 4).

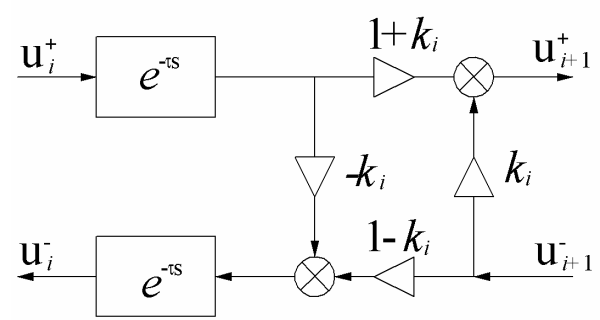

\section{Fig. 4 Functional chart of the single unit in the chain} model

Considered above theory relates to the internal units of the chain model. To build the complete model it is necessary to define boundary conditions at the ends of chain. From the practical point of view the most suitable is to move coordinate system to the left end of the pipeline. There are many approaches to bridging the first and the last unit in chain [5]. The most of these approaches have similar results which base on two hypotheses. The first hypothesis is build on an idea of equality of direct and return waves at the output of the chain

$$
u_{0}^{+}(t-\tau)=u_{0}^{-}(t+\tau)
$$

The second hypothesis is build on condition of matched impedance at the origin of the waves

$$
\begin{aligned}
& u_{p-1}^{+}(t+\tau)=k_{p} \cdot u_{p-1}^{-}(t-\tau)+ \\
& +\left(1+k_{p}\right) u_{p}^{+}(t-\tau), \\
& u_{p}^{-}(t+\tau)=\left(1-k_{p}\right) \cdot u_{p-1}^{-}(t-\tau)- \\
& -k_{p} \cdot u_{p}^{+}(t-\tau),
\end{aligned}
$$

Eq. (21) is the same as Eq. (19) for arbitrary unit in the middle of the chain. Equality of Eq. (21) and Eq. (19) allows us to derive wave equations in arbitrary unit

$$
u_{i}^{+}(t-\tau)=\frac{u_{i-1}^{+}(t+\tau)-k_{i} \cdot u_{i-1}^{-}(t-\tau)}{1+k_{i}},
$$




$$
u_{i}^{-}(t-\tau)=\frac{u_{i-1}^{-}(t-\tau)-k_{i} \cdot u_{i-1}^{+}(t+\tau)}{1+k_{i}} .
$$

Eq. (22) possible to transform into equation with the absolute time member in a way of binding of all time records at beginnings of the every unit with the absolute time at the output of the chain

$$
\begin{aligned}
& y_{i}^{+}(t)=y_{i-1}^{+}(t)+k_{i} \cdot y_{i-1}^{-}(t), \\
& y_{i}^{-}(t+\tau)=k_{i} y_{i-1}^{+}(t)+y_{i-1}^{-}(t),
\end{aligned}
$$

Based on Eq. (23) can be expressed other members

$$
\begin{gathered}
\tau=\frac{l}{2 c}, Y_{i}^{ \pm}=c_{i} \cdot u_{i}^{ \pm}\left(t-\tau-t_{i}\right), \\
t_{i}=2(i+1) \cdot \tau,
\end{gathered}
$$

where $Y_{i}^{ \pm}$are transform components which unambiguously model distributed physical property of the model. The wave velocity can be expressed as

$$
c_{i}=\prod_{j=1}^{i}\left(1+k_{j}\right) i, \quad>0 .
$$

Eq. (23) - Eq. (25) have important practical sense. These equations bind properties of wave propagation in the real model with parameters of an autoregressive filter. The purposes for which the filters are used are sometimes the opposite of those envisaged by the classical theory. If the interest centers on the high frequency component, then it is the low-frequency trend which might be regarded as the nuisance component. Often, the trend and the residue are of equal interest. The filter can be used to model the wave propagation processes if sampling interval of the filter will be equal to $\tau$ from Eq. (23).

\section{DIGITAL FILTER ANALYSIS}

In the classical Wiener and Kolmogorov theory of signal extraction [6] and [7], it is envisaged that the data are generated by a stationary stochastic process and that they form a lengthy sequence. Structure of wave is often depicted as a combination of frequencies, with a low-frequency spectrum, and a noise process, with a high-frequency spectrum. It envisaged that the noise process has a uniform spectrum. This is appropriate whenever the signal process is obscured by a sequence of independently and identically distributed errors of observation. In that case, the noise is aptly described as a nuisance component.
The task of adapting classical signal-extraction filters to limited samples from nonstationary processes has caused difficulties. Problems often arise from not knowing how to supply the initial conditions with which to start a recursive filtering process. By choosing inappropriate starting values, one can generate so-called transient effects which are liable, in fact, to affect all of the processed values. One common approach to the problem of the start-up conditions relies upon the ability to extend the sample by forecasting and back casting [8]. The additional extra-sample values can be used in a runup to the filtering process wherein the filter is stabilized by providing it with a plausible history, if it is working in the direction of time. An approach to the start-up problem is to estimate the requisite initial conditions.

To avoid the start-up problem we apply the filter, in the first instance, to a version of the data sequence which has been reduced to stationarity by repeated differencing. We can proceed to find an estimate of the residual sequence by cumulating its differenced version. If the residual sequence has a significant degree of serial dependence, we can profit from some carefully estimated start-up values to set the process of accumulation in motion. However, if the residual sequence is generated by a weak serial dependence, then it may be acceptable to replace these start-up values by their unconditional expectations which are zeros.

From the theory of digital filters we can derive a transfer function of the direct filter of $p$-order

$$
A_{p}(z)=\sum_{i=0}^{p} a_{p i} z^{-i}
$$

where $a_{p 0}=1$.

The transfer function of the inverse filter is

$$
B_{p}(z)=\sum_{i=0}^{p+1} b_{p i} z^{-i}
$$

where $b_{p}=b_{p+1}=1$.

Based on Eq. (26) and Eq.(27) can be expressed parametric binding of the direct and inverse filters [9]

$$
\begin{aligned}
& A_{p}(z)=A_{p-1}(z)+k_{p} B_{p-1}(z) \\
& z B_{p}(z)=k_{p} A_{p-1}(z)+B_{p-1}(z) .
\end{aligned}
$$

Initial conditions of Eq. (28) are

$$
A_{0}(z)=1, z B_{0}(z)=1 \text {. }
$$


We can notice that Eq. (28) and Eq. (23) structurally are similar. Therefore when the number of units in the chain model is equal to the filter's order then members of Eq. (23) can be calculated from analysis of the signal at the output of the real model by means of direct filter. Moreover if arbitrary signal $e(t)$ effect at the origin of the chain model with its transform $E(z)$ then at the output of the model

$$
Y(z)=\frac{E(z)}{A(z)}
$$

From Eq. (30) can be expressed the transform of signal at the origin of the chain model

$$
E(z)=A(z) y(z) .
$$

Eq. (31) is more interesting from a practical point of view because it allows modeling the filter with finite impulse response by means of Eq. (26). Eq. (30) allows only modeling the filter with infinite impulse response. Structural flowchart of the $A(z)$ and $1 / A(z)$ filters are shown on the Fig. 2 and Fig. 3 respectively. Other way to build the filters with transfer function based on Eq. (28) and lattice structure are shown on Fig. 4 and Fig. 5.

\section{CONCLUSION}

From the shown above becomes clear that the digital filters can be used to represent distributed processes in the chain model. Physical properties of the unit are unambiguous bridged with properties of the real model at the interval which equals to length of one unit.

Using lattice digital filters is more preferable because the signals propagating in cells of the filter are orthogonal. Orthogonally means that amount of cells in the lattice filter do not have influence on the signal.

With this approach change of properties of separate part of the model can be easily model by a way of transferring from constant values of filter coefficients

$$
\left\{\begin{array}{l}
a_{i}(t)=\text { const }, \\
k_{i}(t)=\text { const },
\end{array}\right.
$$

where $i=1,2, \ldots, \mathrm{p}$

to variable value of filter coefficients

$$
\left\{\begin{array}{l}
a_{i}(t)=\mathrm{var}, \\
k_{i}(t)=\mathrm{var},
\end{array}\right.
$$

where $i=1,2, \ldots, p$.

Members of Eq. (33) have own pattern of change which depend on developing and accumulating defects in every unit.

\section{REFERENCES}

[1] Discrete nonlinear systems. Redaction Y. Topchev. Mashinostroenie. Moskow, 1982. p. 176 (in Russian).

[2] S. Kydriakov. Authentication of models of the linear discrete control systems. ЛИАП. Leningrad, 1988. p. 52-56 (in Russian).

[3] N. Zhykovsky. About a water-hammer in waterpipes. Gostechizdat. Moscow, 1949. p. 103 (in Russian).

[4] L. M. Brekhovskikh, V. Goncharov, Mechanics of continua and wave dynamics, Springer-Verlag, Berlin, 1985.

[5] D. Royer, E. Dieulesaint, Elastic waves in Solids I, Springer Verlag, 2000.

[6] A.N. Kolmogorov. Interpolation and Extrapolation, Bulletin de l'academie des sciences de U.S.S.R., Ser. Math., 5. 1941. p. 314.

[7] N. Wiener. Extrapolation, Inter-polation and Smoothing of Stationary Time Series, MIT Technology Press and John Wiley and Sons, New York, 1950. p. 187.

[8] D.S.G. Pollock. Trend estimation and detrending via rational square wave filters. Journal of Econometrics, 99, (2000). p. 317-334.

[9] N. J. Fliege, Multirate Digital Signal Processing, John Wiley \& Sons Ltd. Chichester, 1994.

Igor Lyutak was born in Ivano-Frankivsk, Ukraine in

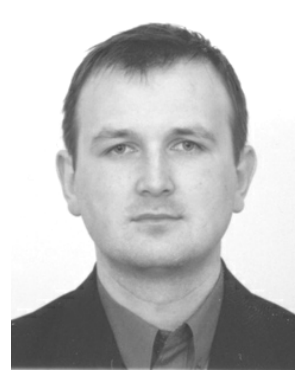
1977. He received the Physical and Engineering degree from the Ivano-Frankivsk National Technical University of Oil and Gas, Ukraine in 1999. In 2002 he received a $P h D$ degree in technical sciences. His current research interests include topics such as non destructive testing of oil-and-gas equipment, developing ultrasonic testing methods and devices. 